\title{
Investigation of Human Mesenchymal Stromal Cells Cultured on PLGA or PLGA/Chitosan Electrospun Nanofibers
}

Fatemeh Ajalloueian ${ }^{1,2}$, Moa Fransson ${ }^{3}$, Hossein Tavanai ${ }^{1}$, Mohammad Massumi ${ }^{4}$, Jöns Hilborn ${ }^{2}$, Katarina Leblanc ${ }^{5}$, Ayyoob Arpanaei $^{6 *}$ and Peetra U Magnusson ${ }^{3^{*}}$

${ }^{1}$ Department of Textile Engineering, Center of Excellence in Applied Nanotechnology, Isfahan University of Technology, Isfahan, 84156-83111, Iran

${ }^{2}$ Polymer Chemistry, Department of Chemistry, Ångström Laboratory, SciLife Lab, Uppsala University, 75121 Uppsala, Sweden

${ }^{3}$ Department of Immunology, Genetics and Pathology, Uppsala University, 75185 Uppsala, Sweden

${ }^{4}$ Department of Physiology, University of Toronto, Toronto, ON M5S 1A8, Canada

${ }^{5}$ Department of Clinical Immunology and Transfusion Medicine, Karolinska Institutet and Hematology Center at Karolinska University Hospital, Stockholm, Sweden

${ }^{6}$ Department of Industrial and Environmental Biotechnology, National Institute of Genetic Engineering and Biotechnology, Tehran, 14965-161, Iran

\begin{abstract}
We compared the viability, proliferation, and differentiation of human Mesenchymal Stromal Cells (MSC) after culture on poly(lactic-co-glycolic acid) (PLGA) and PLGA/chitosan (PLGA/CH) hybrid scaffolds. We applied conventional and emulsion electrospinning techniques, respectively, for the fabrication of the PLGA and PLGA/ $\mathrm{CH}$ scaffolds. Electrospinning under optimum conditions resulted in an average fiber diameter of $166 \pm 33 \mathrm{~nm}$ for the PLGA/CH and $680 \pm 175 \mathrm{~nm}$ for the PLGA scaffold. The difference between the tensile strength of the PLGA and PLGA/CH nanofibers was not significant, but PLGA/CH showed a significantly lower tensile modulus and elongation at break. However, it should be noted that the extensibility of the PLGA/CH was higher than that of the nanofibrous scaffolds of pure chitosan. As expected, a higher degree of hydrophilicity was seen with PLGA/ $\mathrm{CH}$, as compared to PLGA alone. The biocompatibility of the PLGA and PLGA/CH scaffolds was compared using MTS assay as well as analysis by scanning electron microscopy and confocal microscopy. The results showed that both scaffold types supported the viability and proliferation of human MSC, with significantly higher rates on PLGA/ $\mathrm{CH}$ nanofibers. Nonetheless, an analysis of gene expression of MSC grown on either PLGA or PLGA/CH showed a similar differentiation pattern towards bone, nerve and adipose tissues.
\end{abstract}

Keywords: Electrospinning; PLGA; Chitosan; Synthetic-natural scaffold; Mesenchymal stromal cells; Viability; Proliferation

\section{Introduction}

Mesenchymal Stromal Cells (MSC) are considered as a heterogenous cell source for tissue engineering approaches and have received a great deal of attention during the past decade [1-3]. MSC have the capacity to replicate in an undifferentiated state, as well as the potential, when stimulated, to develop into distinct mesodermal tissues, including bone, cartilage, fat, tendon, muscle, and other lineages, both in vitro and in vivo. Although several sources of adult stem cells have been described, bone marrow is the most widely utilized as a source of autologous MSC in the clinic. These multipotent stem cells can give rise to differentiated cells found in adult tissues, have proliferative potential, and facilitate immunomodulatory effects $[3,4]$.

One of the most exciting applications of stem cells is their potential for use in regenerative medicine [5,6]. Autologous human MSC have previously been used in combination with artificial engineered scaffolds in attempts to regenerate new tissues or organs [7]. In order to develop tissue engineered scaffolds that are suitable for preclinical and clinical applications, it is necessary to investigate the biocompatibility of the biomaterial and its capacity to support the lineage/tissue-specific differentiation of stem cells. Several studies have indicated that threedimensional (3D) synthetic scaffolds are suitable for stem cell-based tissue engineering applications $[8,9]$, but stem cell differentiation and organization can be influenced by the scaffold architecture. An ideal implantable scaffold is expected to recapitulate many features of the native Extracellular Matrix (ECM) in the target tissue, in addition to topographical and biochemical cues. Electrospinning is one of the most adopted techniques with the capability of fulfilling such requirements. Electrospun nano/microfibers could not only mimic the ECM 3D structural organization but also make use of a high surface area-to- volume ratio that provides cells with abundant area for attachment, migration, and proliferation $[10,11]$.

Thus far, both synthetic biodegradable materials such as PLA (polylactic acid), PGA (polyglycolic acid), PLGA (poly(lactic-coglycolic acid)), and PCL (polycaprolactone) and naturally derived materials such as fibrin, gelatin, chitosan, and collagen have been well used in tissue engineering and regeneration [12-16]. Synthetic materials usually suffer from poor cell-scaffold attachment interactions because of their hydrophobic structure [17], although they are biodegradable and have good mechanical properties. On the other hand, naturally derived polymers suffer from poor mechanical properties $[18,19]$, despite their biocompatibility and favorable support for cell-scaffold interactions. Therefore, a polyblend of synthetic/natural polymers is of interest for experimental investigations involving stem cells.

In the present study, a polyblend nanofibrous scaffold of PLGA/ chitosan (PLGA/CH) was produced through emulsion electrospinning $[20,21]$, and the adhesion, growth, and differentiation properties of

*Corresponding authors: Ayyoob Arpanaei, Department of Industrial and Environmental Biotechnology, National Institute of Genetic Engineering and Biotechnology, Tehran 14965-161, Iran, Tel: 45-452-549-29; E-mail: aa@nigeb.ac.ir

Peetra U Magnusson, Department of Immunology, Genetics and Pathology, Uppsala University, 75185 Uppsala, Sweden, E-mail: peetra.magnusson@igp.uu.se

Received May 22, 2015; Accepted June 12, 2015; Published June 16, 2015

Citation: Ajalloueian F, Fransson M, Tavanai H, Massumi M, Hilborn J, et al. (2015) Investigation of Human Mesenchymal Stromal Cells Cultured on PLGA or PLGA Chitosan Electrospun Nanofibers. J Bioprocess Biotech 5: 230 doi:10.4172/21559821.1000230

Copyright: (c) 2015 Ajalloueian F, et al. This is an open-access article distributed under the terms of the Creative Commons Attribution License, which permits unrestricted use, distribution, and reproduction in any medium, provided the original author and source are credited. 
human MSC seeded onto this scaffold were compared to those of PLGA electrospun scaffolds. PLGA is a biodegradable polymer with excellent mechanical properties and processability which is used in most of FDA approved therapeutic devices. PLGA electrospun scaffolds have shown mechanical stability to withstand implantation and to support the regeneration of new tissue [22-24]. However, PLGA is a synthetic polymer (with a hydrophobic nature) that suffers from a low affinity for cell attachment. It has been shown that introducing naturally derived polymers such as collagen [24], gelatin [25], or chitosan [26] to PLGA can introduce cell recognition sites and/or improvement of protein adsorption to the scaffold surface to induce better interaction between cells and scaffold. Here, chitosan has been selected as the natural polymer to enhance the biological behavior of the PLGA scaffold. This naturally occurring, biocompatible, biodegradable, and non-toxic polysaccharide is a cationic polymer with a structural similarity to various Glycosaminoglycans (GAGs) of the ECM [27]. Specific interactions between GAGs and growth factors, receptors, and adhesion proteins suggest that the bioactivity of chitosan is a result of its analogous structure to GAGs [28].

Chitosan has been widely used in research for many tissue engineering applications, especially in combination with MSC for bone [28-30] (enhancing osteogenesis) and neural tissue engineering [31,32]. Previous studies have shown that MSC cultured on nonfibrous scaffolds composed of PLGA and chitosan facilitate cell viability, proliferation, and adhesion [31-33]. Considering the key role of autologous human MSC in combination with synthetic scaffolds in regenerative medicine, our aim in this study was to evaluate whether an electrospun polyblend of PLGA/CH, when seeded with human MSC, would demonstrate cell-scaffold superior interactions to those of scaffolds electrospun from PLGA alone.

\section{Materials and Methods}

\section{Reagents}

Chitosan $(\mathrm{MW} \approx 200,000 \mathrm{~g} / \mathrm{mol}$, degree of deacetylation $\approx 90 \%)$ and PLGA (MW 66,000-107,000 g/mol, L-lactide/glycolide, 75:25) were purchased from Sigma-Aldrich (Munich, Germany). Polyvinyl alcohol (PVA, MW $\approx 72,000 \mathrm{~g} / \mathrm{mol}$ ), 1,1,1,3,3,3-hexafluor-2-propanol (HFP), hexamethyldisilazane (HMDS), and glacial acetic acid were obtained from Merck KGaA (Darmstadt, Germany). All solvents were used as received without further purification. Paraformaldehyd (PFA, 1\%) was purchased from Sigma (St. Louis, MO, USA) and CellTiter 96 AQueous One solution was purchased from Promega (Madison, WI, USA).

\section{Fabrication of electrospun PLGA and PLGA/CH scaffolds}

Two different scaffolds of PLGA and PLGA/CH were fabricated in the present study. A PLGA solution $(16 \% \mathrm{w} / \mathrm{v})$ was prepared by dissolving PLGA in $\mathrm{HFP}$ at $50^{\circ} \mathrm{C}$, with magnetic stirring for $3 \mathrm{~h}$. PLGA/CH scaffolds were electrospun from emulsions of PLGA/CH/ PVA. In brief, PLGA was dissolved in HFP $(16 \% \mathrm{w} / \mathrm{v})$, chitosan $(4 \%$ $\mathrm{w} / \mathrm{v})$ was dissolved in acetic acid (14\%) at room temperature, and a PVA solution $(8 \% \mathrm{w} / \mathrm{v})$ was prepared by dissolving PVA (emulsifier) in double-distilled water at $90^{\circ} \mathrm{C}$. The electrospinning emulsion was prepared by adding PVA and chitosan solutions to PLGA solution, followed by mixing with a magnetic stirrer. The volume ratio of the 3 solutions was $1: 1: 1$. The final mixture was stirred at room temperature for $12 \mathrm{~h}$ to obtain a homogenous emulsion. Electrospinning of the prepared emulsion was carried out, with a voltage of $14-16 \mathrm{kV}$ applied to the blunt needle (21-gauge) tip of the $1-\mathrm{mL}$ syringe (filled with the solution/emulsion) and the grounded aluminum foil collector.
The feed rate was $1 \mathrm{~mL} / \mathrm{h}$ for PLGA and $0.25 \mathrm{~mL} / \mathrm{h}$ for PLGA/CH/ PVA. The needle tip-to-collector distance was $15 \mathrm{~cm}$ for both of the scaffolds. Nanofibers collected on aluminum foil were dried overnight under vacuum and then used for the characterization and cell culture experiments. To obtain PLGA/CH nanofibers, PVA was removed from final composites of PLGA/CH/PVA using an aqueous solution containing $50 \%$ ethanol. Weight reduction measurements showed that $8 \mathrm{~h}$ was sufficient for the PVA removal.

\section{Morphology and characterization of the electrospun nanofibers}

The morphology of the electrospun nanofibers was studied with the help of scanning electron microscope (SEM) (Seron Technologies AIS 2100, Seron Technologies Inc., Gyeonggi-do, Korea) micrographs. The average diameter of the electrospun nanofibers was measured by applying Image J software (Image J, National Institutes of Health, USA) to the SEM micrographs. Statistical analysis was carried out using SPSS 8.0. (SPSS Inc. Chicago, IL, USA).

The hydrophilic/hydrophobic nature of the electrospun nanofibrous scaffolds was measured by sessile-drop water-contact angle measurement using a video-based optical contact angle meter (Data Physics OCA 15EC). A $6 \mu \mathrm{L}$ drop of distilled water was placed on the sample surface. The contact angles on the left and right sides of the drop were measured by SCA software. The average of ten angles is reported for each sample.

The tensile properties of the electrospun nanofibrous scaffolds were determined under both dry and wet conditions using a tensile tester (Instron 3345, Canton, MA, USA), with a load cell capacity of $10 \mathrm{~N}$. Test specimens, $10 \mathrm{~mm}$ in width $\times 60 \mathrm{~mm}$ in length, with a thickness of 70-80 $\mu \mathrm{m}$, were tested at a crosshead speed of $10 \mathrm{~mm} / \mathrm{min}$ and a gauge length of $20 \mathrm{~mm}$, under ambient conditions. A minimum of six specimens of each individual scaffold were tested. Wet specimens were prepared by soaking the samples in Phosphate-Buffered Saline (PBS) for $8 \mathrm{~h}$ at $37^{\circ} \mathrm{C}$

The porosity of the PLGA and PLGA/CH fibrous scaffolds was determined using an AutoPore III mercury porosimeter (Micromeritics Instrument, Norcross, GA, USA), based on the total pore volume measured with the mercury intrusion technique.

\section{Isolation and culture of bone marrow-derived MSC}

MSC were isolated and expanded from Bone Marrow (BM) of healthy donors as previously described [34] following approval by the ethics committee at Huddinge University Hospital and thereafter cultured and utilized at Uppsala University (EPN Uppsala, Dnr2013/410). The release criteria for MSC was based on spindle shaped morphology, cell viability $>95 \%$ and flow cytometry of cells with $>95 \%$ positivity for CD73, CD 90 , CD105, HLA-ABC and $<5 \%$ for CD14, CD31, CD34, CD45 and HLA-DR as previously described [34]. The cultures were negative for bacteria, fungi and Polymerase Chain Reaction (PCR)-negative for Mycoplasma pneumoniae. The MSC were cultured in MSC medium consisting of Dulbecco's modified Eagle's medium-low glucose (DMEM-LG), supplemented with $10 \%$ heatinactivated fetal bovine serum (FBS from PAA Laboratories $\mathrm{GmbH}$, Pasching, Austria). In this study, MSC in passages 5-8 from two different donors were used.

\section{Mesenchymal stem cell culture on scaffolds}

Prior to seeding, the nanofibers were sterilized by ultraviolet irradiation of both top and bottom surfaces in a laminar flow hood 
(each side for $20 \mathrm{~min}$ ). To avoid detaching the scaffold from the glass into the medium, sterile plastic rings were used on each scaffold, and $0.5 \mathrm{~mL}$ of culture medium was added to each well (including scaffolds) of a 24 -well plate; the plate was then incubated at $37^{\circ} \mathrm{C}$ overnight. The next day, the medium on the scaffold surface was removed, and MSC suspended in $100 \mu \mathrm{L}$ of culture medium were added to each scaffold at a density of 25,000 cells $/ \mathrm{cm}^{2}$. Cells were incubated with the scaffolds for $2 \mathrm{~h}$, and then an additional $1 \mathrm{~mL}$ of culture medium was added to each well. The incubation of the cells on the scaffolds was then continued as indicated.

\section{Cell and scaffold morphology}

Morphological studies (by SEM) of MSC grown on electrospun PLGA and PLGA/CH nanofibers were performed after 1, 4, and 7 days of cell culture. Cell-seeded nanofiber constructs were harvested and washed with PBS, then fixed in $2.5 \%$ glutaraldehyde for $3 \mathrm{~h}$. The scaffolds were dehydrated with increasing concentrations of ethanol $(30 \%$, $50 \%, 70 \%, 90 \%$ and $100 \%$ ) for 10 min each. Finally, the cell-scaffold constructs were treated with hexamethyldisilazane (HMDS; Fluka Chemical, Milwaukee, WI, USA) to allow further water extraction. The dehydrated, cell-seeded constructs were maintained in desiccators equipped with a vacuum for overnight drying. After sputter-coating with platinum, SEM was used to observe cell and scaffold morphology and cell attachment to the nanofiber scaffolds.

\section{Metabolic activity and proliferation of MSC}

Cell viability and metabolic activity in response to various substrates, Tissue Culture Polystyrene cover slips (TCP), and electrospun samples of PLGA and PLGA/CH, were measured using the MTS cytotoxicity assay (CellTiter 96. AQueous Non-Radioactive Cell Proliferation Assay, Promega, Madison, WI, USA) according to the manufacturer's instructions. The MTS substrate [3-(4,5-dimethylthiazol-2-yl)-5-(3carboxymethoxyphenyl)-2-(4-sulfophenyl)-2H-tetrazolium] is bioreduced by the mitochondria of live cells into a soluble formazan product, which can be quantified by absorbance measurement once it is excreted into the culture medium. Cells were seeded at a density of 15,000 cells per $\mathrm{cm}^{2}$, and the cell activity was evaluated during a 7 -day period. On days 1,4 , and 7, the cell-seeded, electrospun samples were washed with PBS and transferred to new plates containing $500 \mu \mathrm{L}$ of culture medium in each well. Thereafter, $100 \mu \mathrm{L}$ of MTS solution was added to each well. Cells were maintained for an additional $4 \mathrm{~h}$ in a humidified incubator at $37^{\circ} \mathrm{C}$ with $5 \% \mathrm{CO}_{2}$. The absorbance was read at $450 \mathrm{~nm}$ on a Labsystems Multiskan MS plate reader (Labsystems Diagnostics Group, Vantaa, Finland), and the response was defined as (cell-seeded scaffolds A450 mean blank)/(mean TCP 1 day A450 -mean blank) $\times 100 \%$. In this way, the cell activity on each substrate at different time points was compared to that of TCP on day 1 . These assays were done in triplicate, followed by calculation of the mean values and standard deviations.

Cell proliferation was determined by counting the number of DAPI-stained cell nuclei that were associated with the PLGA or PLGA/CH nanofibrous scaffolds. Cell nuclei were counterstained with 4,6-diamidino-2-phenylindole dihydrochloride (DAPI, Sigma-Aldrich; $1 \mu \mathrm{g} / \mathrm{mL}$ ) in three separate samples (PLGA or PLGA/CH) per time point, at 1, 4 and 7 days. A minimum of five randomly selected visual fields were selected and analyzed in a Zeiss 510 confocal microscope (Carl Zeiss AG, Obercochen, Germany).

\section{Cell tracking and confocal analysis of MSC on scaffolds}

MSC grown on electrospun PLGA and PLGA/CH nanofibers were stained with fluorescent 5-chloromethylfluorescein diacetate (CMFDA cell tracker green, Molecular Probes, Life Technologies, Stockholm, Sweden) for observation by fluorescence microscopy of the cells on/ in the scaffolds at 4 and 14 days. In short, cells were incubated prior to the time of examination with $5 \mathrm{mM}$ of CMFDA for $60 \mathrm{~min}$ at $37^{\circ} \mathrm{C}$ in the incubator. The dye was removed after incubation, and the cells were washed with PBS and supplemented with the respective culture medium. The MSC were cultured for an additional $24 \mathrm{~h}$ period, then washed with PBS, fixed in $1 \%$ PFA for $1 \mathrm{~h}$, mounted in mounting medium, and observed with a Zeiss 510 confocal laser scanning microscope (Carl Zeiss).

\section{Quantitative PCR analysis of human MSC}

PLGA and PLGA/CH scaffolds were rinsed with PBS and placed in $500 \mu \mathrm{L}$ of RNALater (Life Technologies, NY, US) for $24 \mathrm{~h}$. Total RNA was extracted using an RNAeasy Micro Kit according to the manufacturer's instructions (Qiagen, Holden, Germany) and transcribed into cDNA with a Superscript II Reverse Transcriptase Kit (Life Technology). Gene-specific polymerase chain reactions were analyzed using the Applied Biosystems StepOne Plus Real-Time PCR Systems machine (Life Technology) during 40 cycles with Applied Biosystems SYBR Green Supermix (Life Technology). All targets (RUNX1, NES, PPARG) were detected with QuantiTect primers (Qiagen GmbH, Hilden, Germany), and the PCR reaction was set up according to the Qiagen QuantiTect protocol. Quantitative values were obtained from the threshold cycle number, and the $\mathrm{x}$-fold change in expression as compared to control samples (MSC grown on tissueculture plastic) was calculated using the threshold cycle number method. Target genes from each individual sample were normalized against a mean threshold cycle number of the target genes SDHA and 18s. Selection of reference target genes was determined by geNorm algorithm analysis and analyzed using qbase plus software (www. biogazelle.com) according to the company's protocol.

\section{Statistical analysis}

Statistical analysis was performed using one-way analysis of variance (ANOVA) and Student's t-test to determine the statistical significance between the two means, evaluated at $\mathrm{p}<0.05$.

\section{Results}

\section{Characterization of the electrospun scaffold}

SEM analysis of PLGA (Figure 1A) and PLGA/CH nanofibers (Figure 1B) demonstrated that PVA removal did not affect the fibrous structure of PLGA/CH nanofibers, and the scaffold kept its integrity, with an average fiber diameter of $166 \pm 33 \mathrm{~nm}$, as compared to a diameter of $680 \pm 175 \mathrm{~nm}$ for the PLGA scaffold. To obtain uniform, bead-free fibers, electrospinning conditions were optimized for both the PLGA solution and PLGA/CH/PVA emulsion. It was observed that the average diameter of nanofibers in the PLGA/CH scaffold was much lower than in the PLGA scaffold.

Water contact-angle measurements of PLGA and PLGA/CH scaffolds demonstrated a large decrease from PLGA $(105 \pm 4.3)$ to PLGA/CH $(27 \pm 8.1)$ nanofibers, revealing the hydrophobic nature of the PLGA material as well as the role of chitosan in conferring hydrophilicity on the PLGA/CH scaffold.

A comparison of the tensile mechanical properties of the PLGA, PLGA/CH/PVA, and PLGA/CH nanofibers (Table 1) revealed that the addition of chitosan and PVA to PLGA led to a strong reduction in the extensibility of the scaffolds, demonstrated as an "elongation 

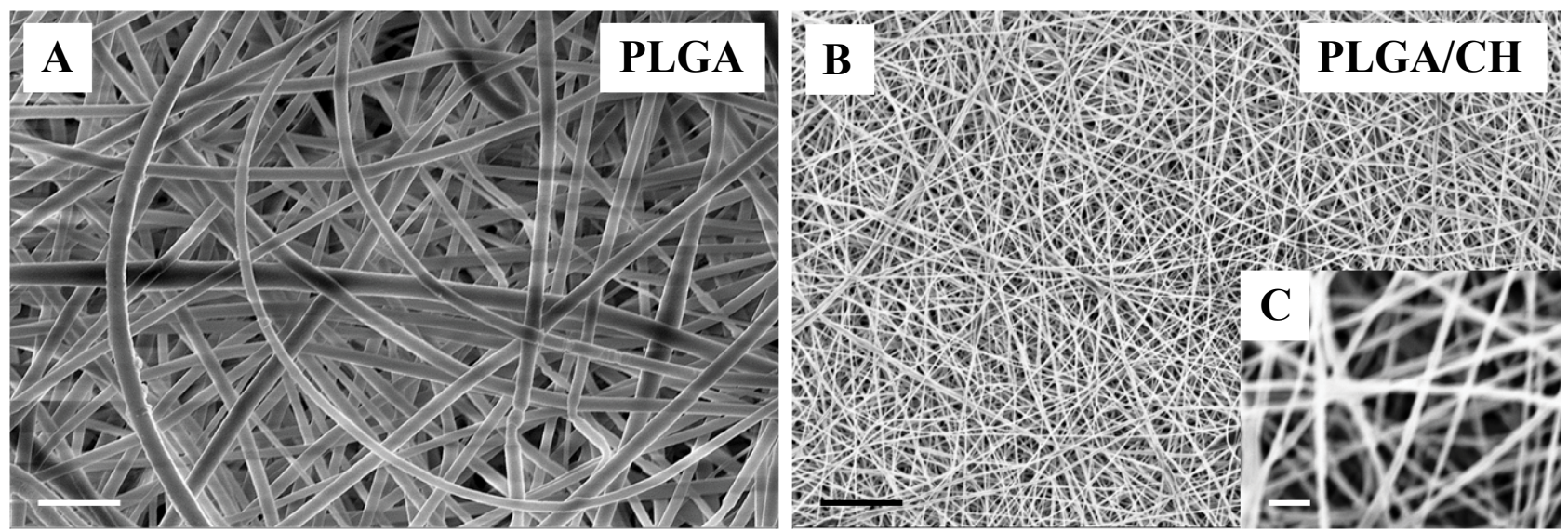

Figure 1: Magnification of the structure of electrospun (A) PLGA and (B and C) PLGA/CH nanofibers visualized by scanning electron microscopy (SEM). Scale bars represent $10 \mu \mathrm{m}$ in $\mathrm{A}, \mathrm{B}$ and $1 \mu \mathrm{m}$ in $\mathrm{C}$.

\begin{tabular}{|c|c|c|c|c|}
\hline & Sample & $\begin{array}{l}\text { Tensile strength } \\
\text { (MPa) }\end{array}$ & $\begin{array}{l}\text { Tensile strain } \\
(\%)\end{array}$ & Modulus (MPa) \\
\hline \multirow{3}{*}{ בิ } & PLGA & $5.6 \pm 1.10$ & $107 \pm 20$ & $354.7 \pm 57.6$ \\
\hline & PLGA/CH/PVA & $5.4 \pm 0.57$ & $14.7 \pm 6.6$ & $296 \pm 32$ \\
\hline & PLGA/CH & $4.3 \pm 0.68$ & $32 \pm 11$ & $75 \pm 10.8$ \\
\hline \multirow{3}{*}{3} & PLGA & $5.35 \pm 0.80$ & $88 \pm 32$ & $227.9 \pm 57.6$ \\
\hline & PLGA/CH/PVA & $4.9 \pm 0.60$ & $12.5 \pm 7.2$ & $211 \pm 24$ \\
\hline & PLGA/CH & $4.14 \pm 1.05$ & $28 \pm 9.4$ & $44 \pm 18.5$ \\
\hline
\end{tabular}

Table 1: Tensile mechanical properties of PLGA, PLGA/CH/PVA, and PLGA/CH scaffolds in dry and wet state.

at break" (dry, from $107 \% \pm 20$ to $14.7 \% \pm 6.6$; wet, from $88 \pm 32$ to $12.5 \pm 7.2)$, whereas there were no significant differences in stiffness (Young's modulus) or tensile strength (maximum tensile stress). Also, PVA removal resulted in a sharp decrease in Young's modulus, from $296 \pm 32 \mathrm{MPa}$ in PLGA/CH/PVA to $75 \pm 10.8 \mathrm{MPa}$ in PLGA/CH under dry conditions. A similar trend was seen for wet samples, from $211 \pm$ $24 \mathrm{MPa}$ to $44 \pm 18.5 \mathrm{MPa}$. However, PVA removal led to an increase in the extensibility of the hybrid structure for both dry $(14.7 \% \pm 6.6$ to $32 \% \pm 11)$ and wet $(12.5 \% \pm 7.2$ to $28 \% \pm 9.4)$ samples. The porosities of the PLGA and PLGA/CH scaffolds were calculated as $77.3 \% \pm 4.6$ and $81.7 \% \pm 2.8$, respectively.

\section{Cell studies}

MSC were cultured on PLGA and PLGA/CH scaffolds and analyzed by SEM after 1, 4 and 7 days (Figure 2). The morphology of the MSC cultured on both scaffolds changed over time. One day after seeding, the MSC had a rounded shape, in contrast to a more elongated cell shape after 4 days (Figure 2), suggesting that the seeded MSC increasingly attached to both PLGA and PLGA/CH nanofibers. Moreover, in continuous culture, the cells covered the substrate to a greater extent (day 7). Length measurements of the cells on PLGA and PLGA/CH showed increased elongation of MSC over time in culture (Figure 3).

To study the proliferation and viability of MSC cultured on the nanofibers, counting of cell nuclei by DAPI staining (Figure $4 \mathrm{~A}$ and 4B, quantification in Figure 4C) and MTS analysis (Figure 4D) were performed after 1, 4, and 7 days of culture. Both PLGA and PLGA/CH scaffolds supported the proliferation of MSC, as shown by the higher count of DAPI-positive nuclei in PLGA/CH nanofibers (Figure 4B, white bars in Figure 4C) than in PLGA nanofibers (Figure 4A, black bars in Figure 4C) and an increased metabolic activity in PLGA/CH scaffolds, as compared to PLGA (Figure 4D, white and black bars, respectively). Significantly higher absorbance was observed after 4 and 7 days when compared to one day of culture in both PLGA and PLGA/ $\mathrm{CH}$ nanofibers (Figure 4D), with higher viability seen in PLGA/CH (Figure 4D, white bars) at all-time points when compared to PLGA (Figure 4D, black bars) and MSC cultured on control tissue-culture plastics (Figure 4D, gray bars).

To examine the morphological changes in MSC seeded onto PLGA and PLGA/CH scaffolds after 4 and 14 days, we cell-tracked the MSC in green (CMFDA) and analyzed them by confocal microscopy (Figure 5). In addition to an increased number of viable cells on PLGA/CH scaffolds as shown in Figure 4, we also found that the MSC elongated over time, making a connected network through interacting filopodia on both PLGA and PLGA/CH scaffolds by 14 days of culture (Figures 3 and 5).

In parallel with our morphologic analysis of MSC after 14 days, we analyzed the differentiation of MSC into different lineages, bone, neuronal and fat by qPCR. An increased expression of bone-(RUNX2), neuro-(NES) and adipose-(PPARG) related genes was detected in cells on both PLGA and PLGA/CH on day 14 when compared to MSC grown on tissue-culture plastic (Figure 6).

\section{Discussion}

Considering the recent attention that has been paid to scaffolds made from mixtures of polymers, especially synthetic-natural polyblends, we have fabricated PLGA/CH nanofibrous scaffolds and compared their behavior to that of PLGA nanofibers. Here we show that by the inclusion of two desired characteristics, the increased strength and durability of the synthetic polymer and the specific cell affinity of the natural one $[18,35,36]$, we have produced an electrospun hybrid with enhanced physical properties and the required biological functionality to support human MSC, when compared to scaffolds made from pure chitosan and PLGA, respectively.

Reports in the literature have noted the potential application of PLGA/CH nanofibrous scaffolds for wound healing and skin tissue reconstruction [37-39]. However, no study has been carried out thus far to investigate the interaction of MSC with this hybrid scaffold. It should be noted that several researchers have focused upon the 
Citation: Ajalloueian F, Fransson M, Tavanai H, Massumi M, Hilborn J, et al. (2015) Investigation of Human Mesenchymal Stromal Cells Cultured on PLGA or PLGA/Chitosan Electrospun Nanofibers. J Bioprocess Biotech 5: 230 doi:10.4172/2155-9821.1000230

day 1

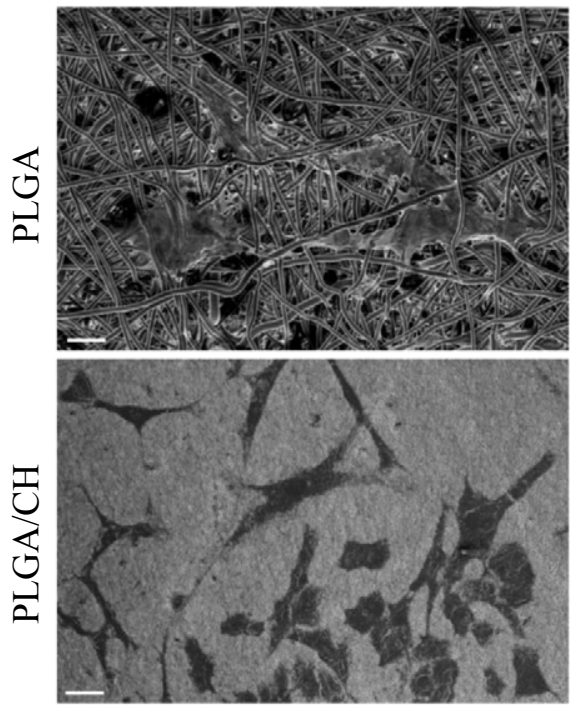

day 4
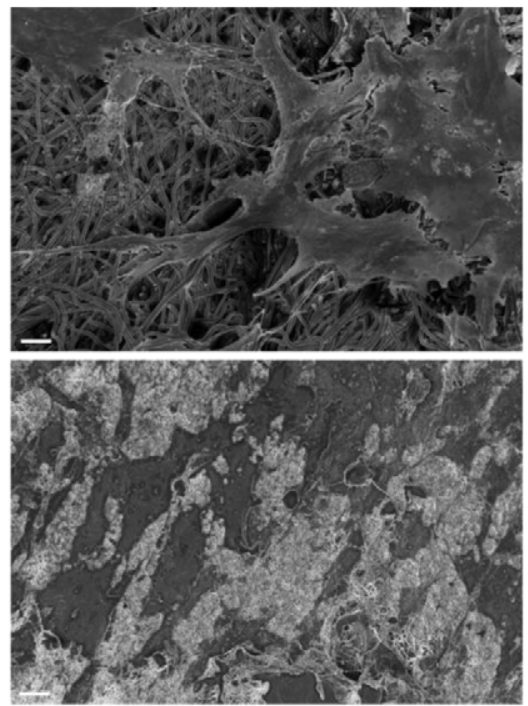

day 7

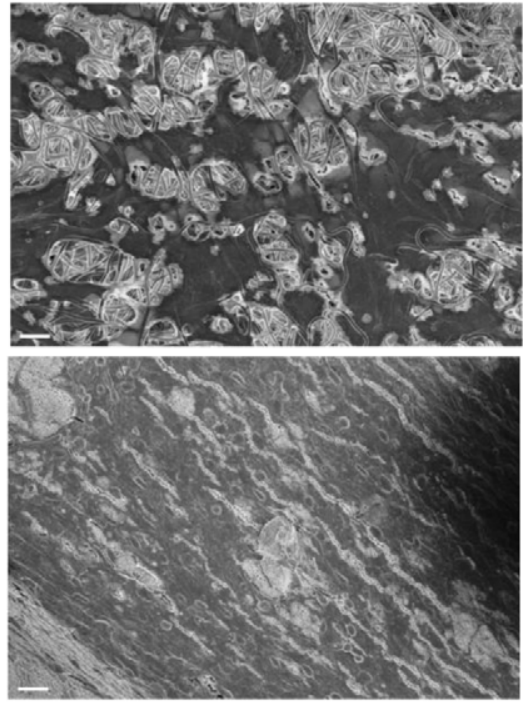

Figure 2: Adhesion of MSC onto electrospun scaffolds under in vitro culturing conditions. MSC were seeded onto PLGA and PLGA/CH scaffolds, and visualized by SEM after 1, 4, and 7 days in culture. MSC adhered, proliferated and stretched out on both of the PLGA and PLGA/CH scaffolds. Scale bars represent $20 \mu \mathrm{m}$.

day 1
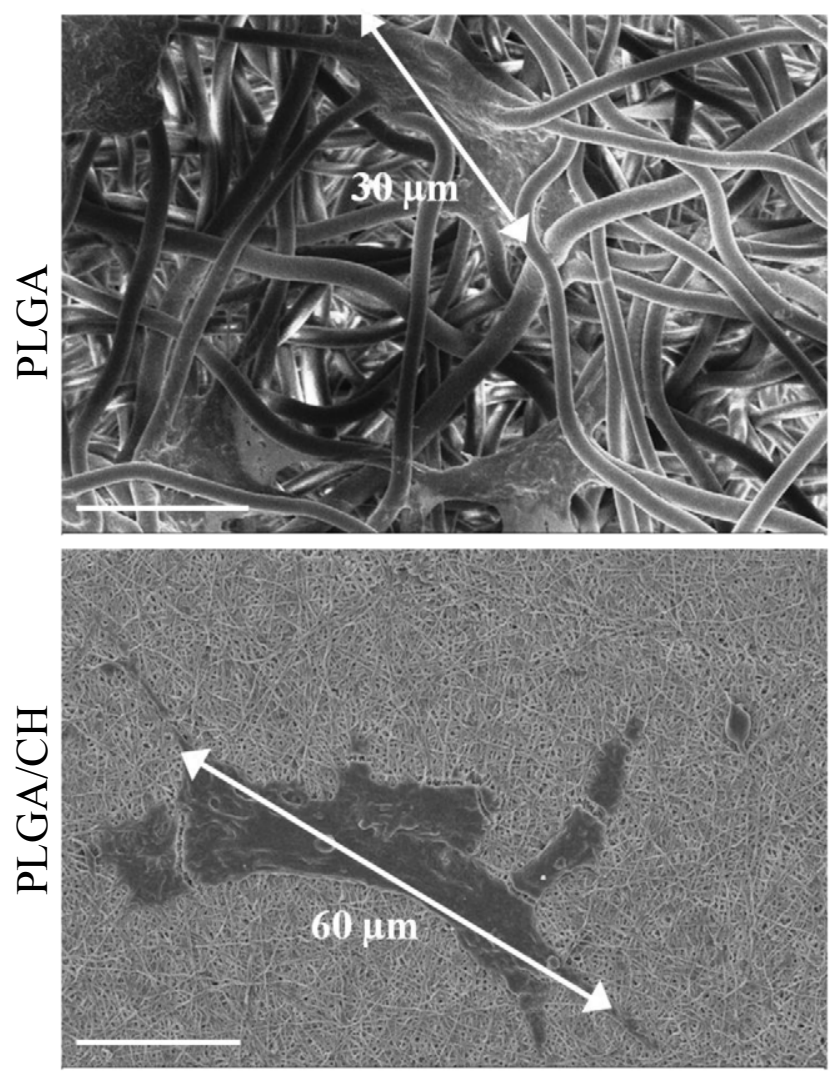

day 14
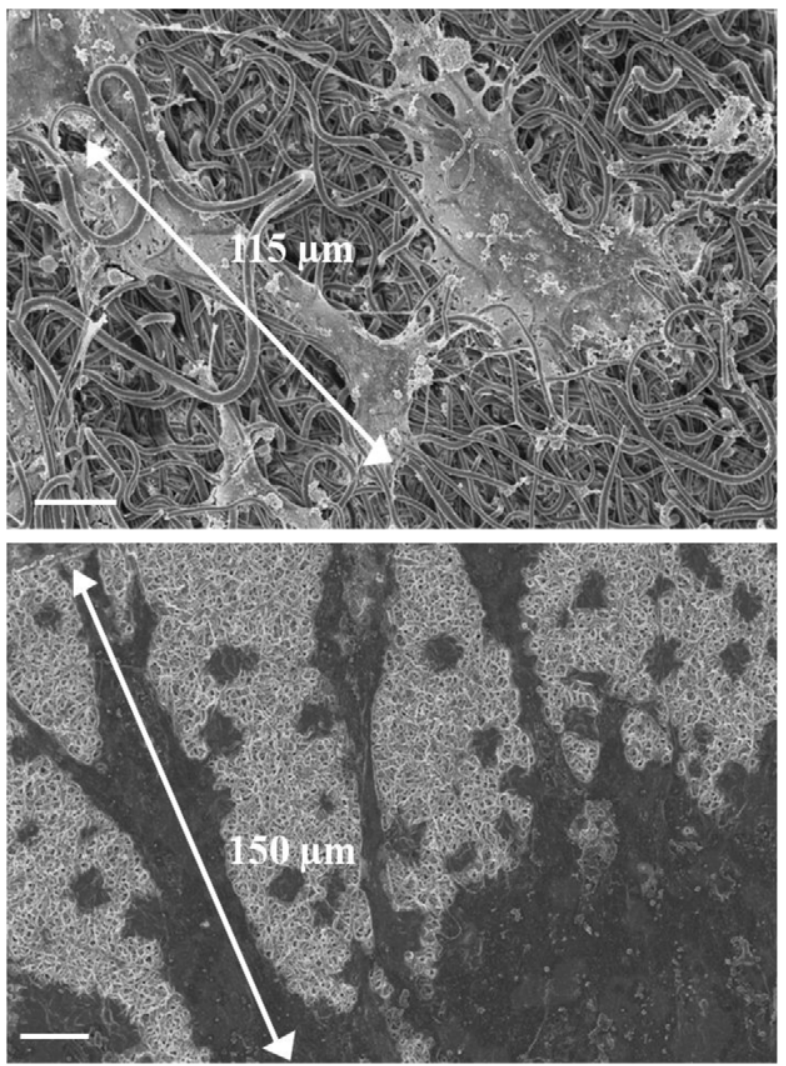

Figure 3: Morphology of MSC seeded onto PLGA and PLGA/CH nanofibers after 1 and 14 days in culture visualized by SEM. 1 day post seeding the MSC have adhered to PLGA and PLGA/CH scaffolds and demonstrate stretched out shape to over $100 \mu \mathrm{m}$ after 14 days in culture. Scale bars represent $20 \mu \mathrm{m}$. 
Citation: Ajalloueian F, Fransson M, Tavanai H, Massumi M, Hilborn J, et al. (2015) Investigation of Human Mesenchymal Stromal Cells Cultured on PLGA or PLGA/Chitosan Electrospun Nanofibers. J Bioprocess Biotech 5: 230 doi:10.4172/2155-9821.1000230
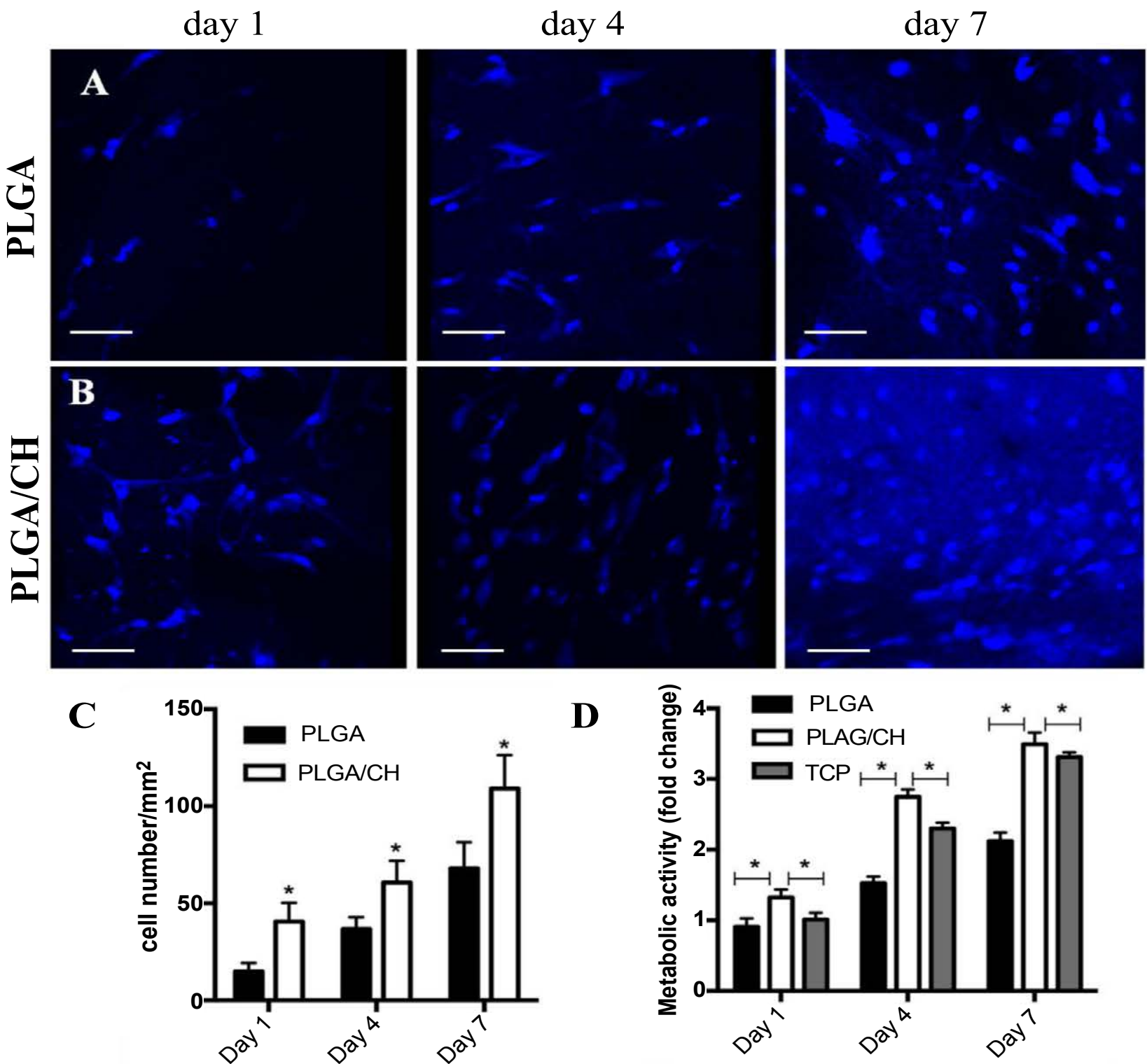

D

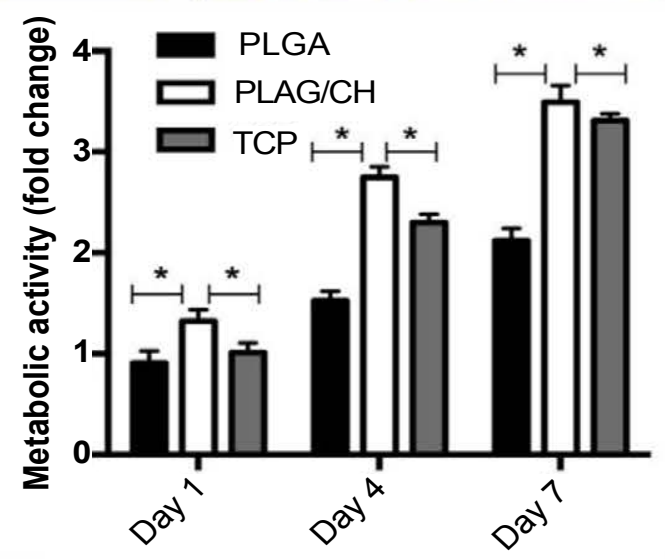

Figure 4: Proliferation and metabolic activity of MSC seeded onto PLGA and PLGA/CH nanofibers. MSC nuclei were stained with DAPI (blue) on (A) PLGA and (B) PLGA/CH scaffolds at 1,4 , and 7 days post seeding. Scale bars represent $100 \mu \mathrm{m}$. (C) Proliferation measured as total cell count of MSC seeded onto PLGA and PLGA/CH nanofibers at 1,4 , and 7 days in culture. Cell proliferation increased up to 7 days post seeding on both PLGA (black) and PLGA/CH (white) scaffolds. The total cell count of MSC on PLGA/CH was significantly higher at all of the investigated time points compared to PLGA ( $p<0.05)$. (D) Metabolic activity measured as a reflection of mitochondrial function using an MTS assay in MSC seeded onto PLGA (black) and PLGA/CH (white) nanofibers and tissue-culture plastic (TCP, gray). Metabolic activity of MSC was significantly increased when growing on PLGA/CH compared to PLGA $(p<0.05)$ and TCP $(p<0.05)$.

interaction between polyblend materials and adult stem cells. They have demonstrated improved attachment and growth of stem cells, enhanced mechanical performance, and increased wettability as compared to scaffolds prepared from either of the individual polymers making up the blend [40]. The interaction of MSC with PLGA/CH scaffolds has also been previously studied [31,33], but the scaffolds applied were not nanofibrous. For instance, Kuo et al. showed that MSC seeded onto PLGA/chitosan scaffolds fabricated by crosslinking and lyophilization were prone to differentiate toward osteoblasts without guidance, and toward neurons after introduction of nerve growth factor (NGF) [31]. In another work, Xue et al. incorporated autologous MSC into a neural scaffold consisting of a chitosan conduit with inserted PLGA filaments
[33]. They showed satisfactory repair and rehabilitation of large gaps after peripheral nerve injury in dogs, suggesting that a combination of autologous MSC with PLGA/CH could be used for neural tissue engineering.

Here, our comparison of PLGA/CH and PLGA nanofibers has demonstrated that PLGA/CH is more hydrophilic than PLGA, that it supports higher rates of cell proliferation, and that it has a mechanical strength similar to that of PLGA. We also demonstrated that both the scaffolds showed high porosities (77.3\% for PLGA and $81.7 \%$ for PLGA/CH), which are characteristic of electrospun scaffolds. The difference between the porosity of two scaffold types was not statistically 
Citation: Ajalloueian F, Fransson M, Tavanai H, Massumi M, Hilborn J, et al. (2015) Investigation of Human Mesenchymal Stromal Cells Cultured on PLGA or PLGA/Chitosan Electrospun Nanofibers. J Bioprocess Biotech 5: 230 doi:10.4172/2155-9821.1000230

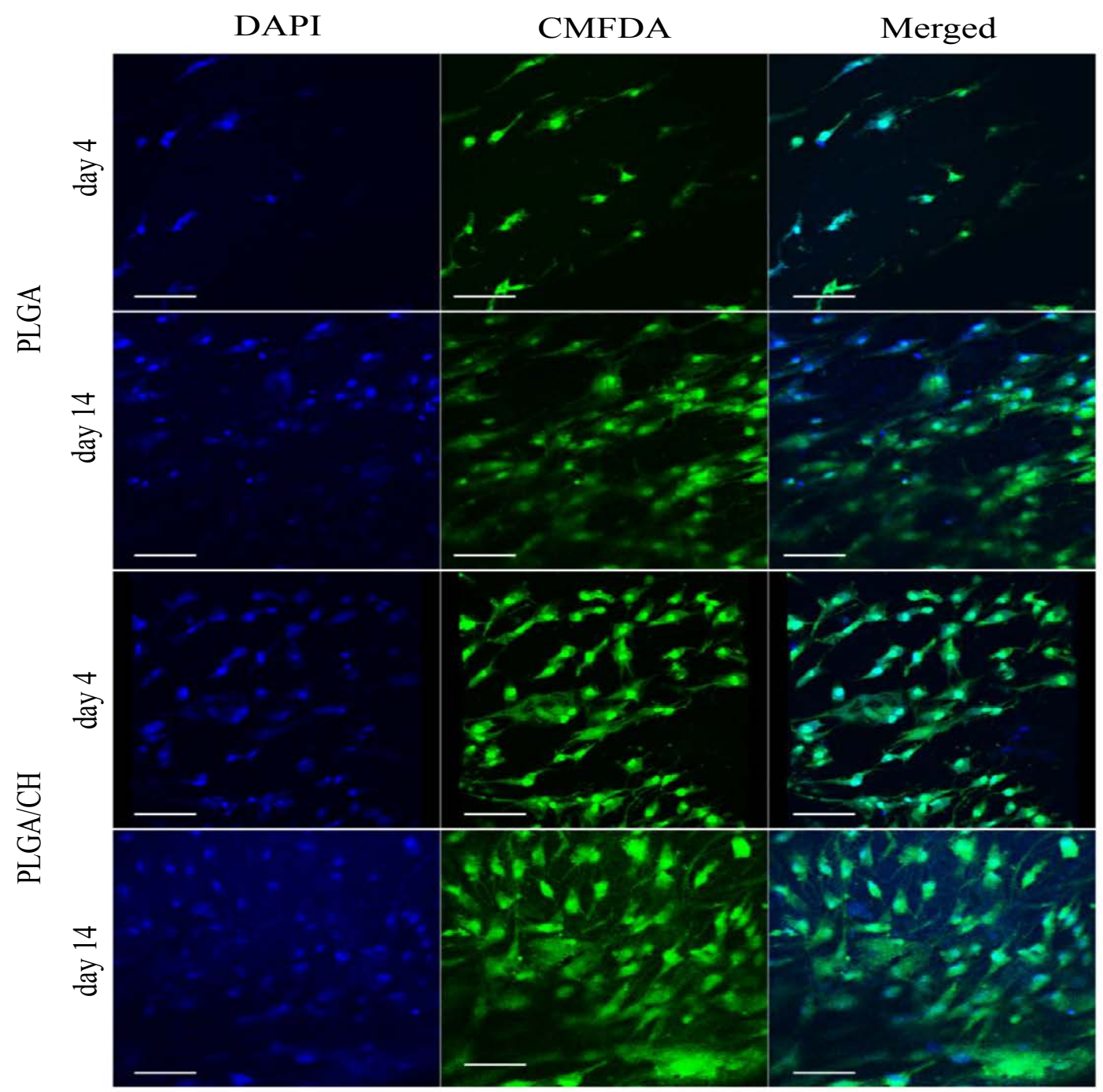

Figure 5: Morphology of MSC seeded onto PLGA and PLGA/CH nanofibers after 4 and 14 days in culture visualized by confocal microscopy. MSC are labeled with a cell tracking dye (CMFDA) here seen in green and nuclei stained with DAPI (blue). At 14 days post seeding the MSC stretched out to over $100 \mu \mathrm{m}$ and the total number of cells covered the surface at a higher density on both nanofibers. Scale bars represent $100 \mu \mathrm{m}$.
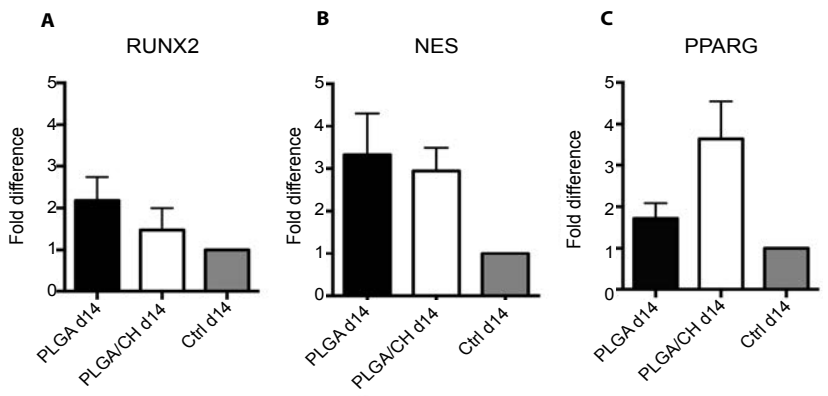

Figure 6: Genetic profile of MSC grown on PLGA and PLGA/CH on day14 $(n=3)$. MSC grown on PLGA and PLGA/CH were analyzed by quantitative PCR for genetic markers in (A) bone (RUNX2): mean-fold difference, PLGA 2.17; PLGA/CH, 1.46; (B) neurological marker (NES): mean-fold difference, PLGA, 3.33; PLGA/CH, 2.94; and (C) fat (PPARG): mean-fold difference, PLGA, 1.72; PLGA/CH, 3,63, when each was compared to MSC grown on tissue-culture plastic. significant. It should be noted that our final construct contained $80 \%$ PLGA and 20\% chitosan. This ratio was selected because of the role of PLGA as a mechanical support (the main part of the construct) and chitosan as the agent to improve the cell-scaffold interaction (used in a lower ratio). As mentioned earlier, such hybrid scaffolds are expected to demonstrate improved mechanical properties over those made from the natural polymer only. According to previous studies, chitosan nanofibers undergo a sharp decrease in mechanical strength and Young's modulus when hydrated [41]. This property limits their biomedical application, given that specimens are kept in culture and/ or implanted in the body. However, the trends seen in the variation in mechanical properties of chitosan nanofibers under dry and wet conditions were not seen for our hybrid scaffold. As shown in Table 1, only a small decrease in strength and stiffness was seen for the PLGA/ $\mathrm{CH}$ scaffold when dry and wet conditions were compared. Moreover, the difference in tensile strength between PLGA/CH and PLGA was almost insignificant. 
It should also be noted that PVA extraction resulted in an increase in the extensibility of the hybrid structure. This increase could be a result of the longitudinal shrinkage of $\sim 30 \%$ that was seen after PVA extraction; the increase provides an advantage for the PLGA/CH scaffold over chitosan nanofibrous scaffolds, which show only very little elongation at the break [41,42]. Taken together, our findings suggest that, given its improved mechanical properties when compared to chitosan nanofibers and strength similar to that of our PLGA scaffold, the hybrid PLGA/CH scaffold can be considered an appropriate candidate for biomedical and tissue engineering applications.

Our biocompatibility studies showed that both the PLGA and PLGA/CH scaffolds supported cell proliferation after 1, 4, and 7 days of culture. However, the increased metabolic activity and proliferation of MSC with PLGA/CH suggested that the polyblend construct provided more favorable growth and survival conditions for MSC than did PLGA alone. The improved attachment and growth of cells on substrates made from chitosan might be due to the cationic nature of the amine groups in chitosan, which enhances the attraction for negatively charged cells as well [28]. As we grow our MSC in media containing serum it is more likely that cells attach to adsorbed serum proteins. It should be taken into account that having chitosan incorporated into PLGA as a biopolymer to make it more bio-receptive also led to a decrease in the average diameter (and hence increased surface area) of the PLGA/CH nanofibers. Others have also reported a decrease in fiber diameter in polyblends of PLGA/gelatin and PLGA/collagen when compared to PLGA, as a result of adding a biopolymer to PLGA $[43,44]$. In our study, we performed electrospinning under optimal conditions that led to the formation of nanofibers of PLGA and PLGA/ $\mathrm{CH}$ with an average diameter of $680 \mathrm{~nm}$ and $166 \mathrm{~nm}$, respectively. The improved viability and attachment of MSC to PLGA/CH as compared to PLGA could be a function of the higher bioactivity, smaller fiber diameter, and larger surface area [45] of the hybrid construct. There are several studies investigating various nanofibres and their capacity to affect proliferation and function of stem cells [46-48]. Here we have investigated the effect of adding the natural polymer chitosan to PLGA nanofibers, showing increased proliferation and metabolic activity of human MSC most likely to an increase in biofunctionality of the scaffold. Our morphological studies using SEM and confocal microscopy not only confirmed that an increased number of cells reached confluency in 14 days but also showed extension of individual cells on both PLGA and PLGA/CH scaffolds that formed a connected network through interacting filopodia.

Quantitative PCR analysis demonstrated that both PLGA and PLGA/CH nanofibrous scaffolds supported the gene regulation of MSC into bone, fat and neuronal cells. The quantitative PCR results confirmed that the blending and electrospinning of chitosan with PLGA did not hamper the ability of PLGA [49] to promote the differentiation of bone, fat, and neuronal cells from MSC.

\section{Conclusions}

Herein, we have shown that the nanofibrous scaffold composed of PLGA/CH created a favorable substrate supporting the adherence, survival and proliferation of MSC with potential use in tissue engineering. Not only were the tensile strength and porosity similar to those of the electrospun scaffold of PLGA, but we saw increased hydrophilicty and higher viability and proliferation of MSC with the hybrid scaffolds than with PLGA alone. Our quantitative PCR analyses confirmed that both PLGA/CH and PLGA scaffolds could potentially have the ability to direct the MSC phenotype.

\section{Acknowledgments}

This study was supported by grants from EXODIAB (UFV-PA 2012/2330), Stem Therapy, the Swedish Research Council (A0290401, A0290402) and The Swedish Society of Medicine. We thank Dr. Deborah McClellan for editorial assistance.

\section{References}

1. Murphy MB, Moncivais K and Caplan Al (2013) Mesenchymal stem cells: environmentally responsive therapeutics for regenerative medicine. Exp Mol Med 45: e54.

2. Gustafsson $Y$, Haag J, Jungebluth P, Lundin V, Lim ML, et al. (2012) Viability and proliferation of rat MSCs on adhesion protein-modified PET and PU scaffolds. Biomaterials 33: 8094-8103.

3. Ringe J, Kaps C, Burmester GR, Sittinger M (2002) Stem cells for regenerative medicine: advances in the engineering of tissues and organs. Naturwissenschaften 89: 338-351.

4. Hwang NS, Varghese S, Elisseeff J (2008) Controlled differentiation of stem cells. Adv Drug Deliv Rev 60: 199-214.

5. Macchiarini $P$, Jungebluth $P$, Go T, Asnaghi MA, Rees LE, et al. (2008) Clinical transplantation of a tissue-engineered airway. Lancet 372: 2023-2030.

6. Elliott MJ, De Coppi P, Speggiorin S, Roebuck D, Butler CR, et al. (2012) Stemcell-based, tissue engineered tracheal replacement in a child: a 2-year followup study. Lancet 380: 994-1000.

7. Atala A, Bauer SB, Soker S, Yoo JJ, Retik AB (2006) Tissue-engineered autologous bladders for patients needing cystoplasty. Lancet 367: 1241-1246.

8. Levenberg S, Huang NF, Lavik E, Rogers AB, Itskovitz-Eldor J, et al. (2003) Differentiation of human embryonic stem cells on three-dimensional polymer scaffolds. Proc Natl Acad Sci U S A 100: 12741-12746.

9. Hwang NS, Kim MS, Sampattavanich S, Baek JH, Zhang Z, et al. (2006) Effects of three-dimensional culture and growth factors on the chondrogenic differentiation of murine embryonic stem cells. Stem Cells 24: 284-291.

10. Huang ZM, Zhang YZ, Kotaki M, Ramakrishna S (2003) A review on polymer nanofibers by electrospinning and their applications in nanocomposites. Compos Sci Technol 63: 2223-2253.

11. Milleret $V$, Simona $B$, Neuenschwander $P$, Hall $H$ (2011) Tuning electrospinning parameters for production of 3D-fiber-fleeces with increased porosity for soft tissue engineering applications. Eur Cell Mater 21: 286-303.

12. Li M, Mondrinos MJ, Chen X, Gandhi MR, Ko FK, et al. (2006) Co-electrospun poly(lactide-co-glycolide), gelatin, and elastin blends for tissue engineering scaffolds. J Biomed Mater Res A 79: 963-973.

13. Ma X, Che N, Gu Z, Huang J, Wang D, et al. (2013) Allogenic mesenchymal stem cell transplantation ameliorates nephritis in lupus mice via inhibition of B-cell activation. Cell Transplant 22: 2279-2290.

14. Haugh MG, Thorpe SD, Vinardell T, Buckley CT, Kelly DJ (2012) The application of plastic compression to modulate fibrin hydrogel mechanical properties. $J$ Mech Behav Biomed Mater 16: 66-72

15. Ajalloueian F, Zeiai S, Fossum M, Hilborn JG (2014) Constructs of electrospun PLGA, compressed collagen and minced urothelium for minimally manipulated autologous bladder tissue expansion. Biomaterials 35: 5741-5748.

16. Ajalloueian F, Zeiai S, Rojas R, Fossum M, Hilborn J (2013) One-stage tissue engineering of bladder wall patches for an easy-to-use approach at the surgical table. Tissue Eng Part C Methods 19: 688-696.

17. Bhattarai N, Edmondson D, Veiseh O, Matsen FA, Zhang M (2005) Electrospun chitosan-based nanofibers and their cellular compatibility. Biomaterials 26 6176-6184.

18. Bhattarai N, Li Z Gunn J, Leung M, Cooper A et al (2009) Natural-synthetic polyblend nanofibers for biomedical applications. Adv Mater 21: 2792-2797.

19. Gunn J, Zhang M (2010) Polyblend nanofibers for biomedical applications: perspectives and challenges. Trends Biotechnol 28: 189-197.

20. Qi H, Hu P, Xu J, Wang A (2006) Encapsulation of drug reservoirs in fibers by emulsion electrospinning: morphology characterization and preliminary release assessment. Biomacromolecules 7: 2327-2330. 
Citation: Ajalloueian F, Fransson M, Tavanai H, Massumi M, Hilborn J, et al. (2015) Investigation of Human Mesenchymal Stromal Cells Cultured on PLGA or PLGA/Chitosan Electrospun Nanofibers. J Bioprocess Biotech 5: 230 doi:10.4172/2155-9821.1000230

Page 9 of 9

21. Ajalloueian $\mathrm{F}$, Tavanai $\mathrm{H}$, Hilborn $\mathrm{J}$, Donzel-Gargand $\mathrm{O}$, Leifer $\mathrm{K}$, et al. (2014) Emulsion electrospinning as an approach to fabricate PLGA/chitosan nanofibers for biomedical applications. Biomed Res Int 2014: 475280.

22. Shin $\mathrm{HJ}$, Lee $\mathrm{CH}$, Cho IH, Kim YJ, Lee YJ, et al. (2006) Electrospun PLGA nanofiber scaffolds for articular cartilage reconstruction: mechanical stability, degradation and cellular responses under mechanical stimulation in vitro. $J$ Biomater Sci Polym Ed 17: 103-119.

23. Inanç B, Arslan YE, Seker S, Elçin AE, Elçin YM (2009) Periodontal ligament cellular structures engineered with electrospun poly(DL-lactide-co-glycolide) nanofibrous membrane scaffolds. J Biomed Mater Res A 90: 186-195.

24. Nakanishi Y, Chen G, Komuro H, Ushida T, Kaneko S, et al. (2003) Tissueengineered urinary bladder wall using PLGA mesh-collagen hybrid scaffolds: a comparison study of collagen sponge and gel as a scaffold. J Pediatr Surg 38: $1781-1784$

25. Prabhakaran MP, Kai D, Ghasemi-Mobarakeh L, Ramakrishna S (2011) Electrospun biocomposite nanofibrous patch for cardiac tissue engineering. Biomed Mater 6: 055001

26. Wang Q, Jamal S, Detamore MS, Berkland C (2011) PLGA-chitosan/PLGAalginate nanoparticle blends as biodegradable colloidal gels for seeding human umbilical cord mesenchymal stem cells. J Biomed Mater Res A 96: 520-527.

27. Peniche $\mathrm{H}$, Peniche $\mathrm{C}$ (2011) Chitosan nanoparticles: a contribution to nanomedicine. Polym Int 60: 883-889.

28. Moutzouri AG, Athanassiou GM (2011) Attachment, spreading, and adhesion strength of human bone marrow cells on chitosan. Ann Biomed Eng 39: 730-741.

29. Verma D, Katti KS, Katti DR (2010) Osteoblast adhesion, proliferation and growth on polyelectrolyte complex-hydroxyapatite nanocomposites. Philos Trans A Math Phys Eng Sci 368: 2083-2097.

30. Venkatesan J, Kim SK (2010) Chitosan composites for bone tissue engineering -an overview. Mar Drugs 8: 2252-2266.

31. Kuo YC, Yeh CF, Yang JT (2009) Differentiation of bone marrow stromal cells in poly(lactide-co-glycolide)/chitosan scaffolds. Biomaterials 30: 6604-6613.

32. Hu N, Wu H, Xue C, Gong Y, Wu J, et al. (2013) Long-term outcome of the repair of $50 \mathrm{~mm}$ long median nerve defects in rhesus monkeys with marrow mesenchymal stem cells-containing, chitosan-based tissue engineered nerve grafts. Biomaterials 34: 100-111.

33. Xue C, Hu N, Gu Y, Yang Y, Liu Y, et al. (2012) Joint use of a chitosan PLGA scaffold and MSCs to bridge an extra large gap in dog sciatic nerve. Neurorehabil Neural Repair 26: 96-106.

34. Le Blanc K, Tammik L, Sundberg B, Haynesworth SE, Ringdén O (2003) Mesenchymal stem cells inhibit and stimulate mixed lymphocyte cultures and mitogenic responses independently of the major histocompatibility complex. Scand J Immunol 57: 11-20.

35. Wang CY, Zhang KH, Fan CY, Mo XM, Ruan HJ, et al. (2011) Aligned natural- synthetic polyblend nanofibers for peripheral nerve regeneration. Acta Biomater 7: 634-643.

36. Meng ZX, Zheng W, Li L, Zheng YF (2011) Fabrication, characterization and in vitro drug release behavior of electrospun PLGA/chitosan nanofibrous scaffold. Mater Chem Phys 125: 606-611.

37. Xie D, Huang H, Blackwood K, MacNeil S (2010) A novel route for the production of chitosan/poly(lactide-co-glycolide) graft copolymers for electrospinning. Biomed Mater 5: 065016

38. Duan B, Yuan X, Zhu Y, Zhang Y, Li X, et al. (2006) A nanofibrous composite membrane of PLGA-chitosan/PVA prepared by electrospinning. Eur Polym J 42: 2013-2022.

39. Duan B, Wu L, Yuan X, Hu Z, Li X, et al. (2007) Hybrid nanofibrous membranes of PLGA/chitosan fabricated via an electrospinning array. J Biomed Mater Res A 83: 868-878.

40. Zhang Y, Ouyang H, Lim CT, Ramakrishna S, Huang ZM (2005) Electrospinning of gelatin fibers and gelatin/PCL composite fibrous scaffolds. J Biomed Mater Res B Appl Biomater 72: 156-165

41. Wan Y, Cao X, Zhang S, Wang S, Wu Q (2008) Fibrous poly(chitosan-g-DLlactic acid) scaffolds prepared via electro-wet-spinning. Acta Biomater 4: 876-886.

42. Schiffman JD, Schauer CL (2007) Cross-linking chitosan nanofibers. Biomacromolecules 8: 594-601.

43. Prabhakaran MP, Kai D, Ghasemi-Mobarakeh L, Ramakrishna S (2011) Electrospun biocomposite nanofibrous patch for cardiac tissue engineering. Biomed Mater 6: 055001.

44. Jose MV, Thomas V, Dean DR, Nyairo E (2009) Fabrication and characterization of aligned nanofibrous PLGA/Collagen blends as bone tissue scaffolds. Polymer 50: 3778-3785.

45. Chen M, Patra PK, Lovett ML, Kaplan DL, Bhowmick S (2009) Role of electrospun fibre diameter and corresponding specific surface area (SSA) on cell attachment. J Tissue Eng Regen Med 3: 269-279.

46. Chua KN, Chai C, Lee PC, Tang YN, Ramakrishna S, et al. (2006) Surfaceaminated electrospun nanofibers enhance adhesion and expansion of human umbilical cord blood hematopoietic stem/progenitor cells. Biomaterials 27 6043-6051.

47. Martino S, D'Angelo F, Armentano I, Kenny JM, Orlacchio A (2012) Stem cellbiomaterial interactions for regenerative medicine. Biotechnol Adv 30: 338-351.

48. Du J, Tan E, Kim HJ, Zhang A, Bhattacharya R, et al. (2014) Comparative evaluation of chitosan, cellulose acetate, and polyethersulfone nanofiber scaffolds for neural differentiation. Carbohydr Polym 99: 483-490.

49. Massumi M, Abasi M, Babaloo $H$, Terraf $P$, Safi M, et al. (2012) The effect of topography on differentiation fates of matrigel-coated mouse embryonic stem cells cultured on PLGA nanofibrous scaffolds. Tissue Eng Part A 18: 609-620. 